Abstract PWE-180 Table 1 Ability to make a diagnosis for each patient vignette

\begin{tabular}{llll}
\hline & IBS-C & IBS-D & IBD \\
\hline Type of physician & Experts / GEs / GPs & Experts / GEs / GPs & Experts / GEs / GPs \\
Correct diagnosis & $88 \% / 56 \% / 31 \%$ & $92 \% / 72 \% / 64 \%$ & $92 \% / 87 \% / 85 \%$ \\
Incorrect diagnosis & $4 \% / 4 \% / 5 \%$ & $8 \% / 12 \% / 14 \%$ & $4 \% / 13 \% / 14 \%$ \\
Don't know & $8 \% / 40 \% / 64 \%$ & $-/ 16 \% / 22 \%$ & $4 \% /-/ 1 \%$ \\
\hline
\end{tabular}

Introduction The confident diagnosis of chronic abdominal conditions can be challenging. This study assessed the diagnostic process in irritable bowel syndrome with constipation (IBSC), irritable bowel syndrome with diarrhoea (IBSD), inflammatory bowel disease (IBD) and chronic constipation (CC).

Methods Online interviews were conducted with 25 experts, 100 gastroenterologists (GEs) and 104 general practitioners (GPs) from Germany, Spain, France, Italy and the United Kingdom to explore perception, attitude and diagnostic approach to IBS. Physicians were also presented patient vignettes describing a typical case of IBS-C, IBS-D, IBD and CC, respectively. For each vignette, participants were asked to make a diagnosis and to give details on further clinical investigations and management of each case.

Results The CC and IBS-C vignettes caused most diagnostic difficulties. For the IBS-C vignette, most GEs and GPs who did not make a correct diagnosis were unsure of the diagnosis. In contrast, most physicians who did not make a correct diagnosis for the CC vignette gave an incorrect diagnosis of IBS-C.

Physicians' confidence level in their diagnosis was 7.0/9 for the IBS-D vignette, 6.8/9 for IBS-C and 6.7/9 for CC. The score was lowest for IBD (6.3) as most physicians stated they would wait for the results of further investigations prior to diagnosis. Experts were most likely to endorse a positive approach to the diagnosis of IBS, IBD or CC, whereas GEs and GPs preferred to adopt a diagnosis by exclusion approach. For the IBS and CC vignettes, most physicians' next action would be to prescribe treatment. However, for the IBD vignette, the next action would be to conduct tests. Most experts (96\%) and GEs (73\%) claimed to have a good knowledge of the Rome III diagnostic criteria, compared to only $15 \%$ of GPs.

Conclusion The study highlights some of the difficulties experienced by GPs, GEs and experts in diagnosing chronic abdominal conditions. Physicians found differentiating between IBS-C and $\mathrm{CC}$ to be particularly challenging. Diagnostic criteria designed for research purposes may not necessarily be applicable in standard clinical practice.

Study funded by Almirall S. A.

Disclosure of Interest V. Andresen Consultant for: Almirall, Astra Zeneca, Norgine, Shire, Conflict with: Almirall, Abbvie, Aptalis, Ardeypharm, Norgine, Shire, Mundipharma, Falk, P. Whorwell: None Declared, J. Fortea Employee of: Almirall S. A., J. Milce Grant/research support from: Almirall, Consultant for: Kantar Health.

\section{PWE-181 QUANTITATIVE ASSESSMENT OF GLOBAL SMALL BOWEL MOTILITY IN CHRONIC INTESTINAL PSEUDO-OBSTRUCTION AND CONTROLS: A PRELIMINARY STUDY}

${ }^{1}$ SK Butt*, ${ }^{2} \mathrm{~A}$ Menys, ${ }^{2} \mathrm{D}$ Atkinson, ${ }^{2} \mathrm{~A}$ Plumb, ${ }^{2} \mathrm{~S}$ Taylor, ${ }^{1} \mathrm{~N}$ Zarate-Lopez, ${ }^{1} \mathrm{~A}$ Emmanuel. ${ }^{1}$ Gastroenterology, UCL London, UK; ${ }^{2}$ Centre for Medical Imaging, UCL, London, UK
Introduction In this preliminary report, we present the initial results of a prospective investigation comparing MRI quantified global small bowel motility in healthy controls and patients with proven clinical and radiological Chronic Intestinal pseudo-obstruction (CIPO). Diagnosis is initially difficult and often delayed, many patients undergoing unnecessary surgical intervention prior to final diagnosis. MRI offers a potential non-invasive modality of diagnosis and monitoring, employing post-processing quantitation of global metrics describing small bowel motility ${ }^{1}$.

Methods Subject selection: 11 healthy non-smoking volunteers (7 Male, mean age 33[22 to 48]) and 5 CIPO patients (3 Male, mean age $53[32$ to 82$]$ ) were recruited. CIPO patients stopped any medications that influenced small bowel motility for one week prior to scan including opioids, anti-emetics and anti-diarrhoeals. Study overview: Participants underwent a single MRI motility scan before and immediately after an injection of $0.5 \mathrm{mg}$ IV neostigmine, a cholinomimetic with potent prokinetic action. Statistics: Data normality was assessed using Shapiro-Wilk testing. 1) Baseline motility was compared in CIPO patients and controls. 2) Percent change in motility between baseline and post-neostigmine was compared between groups. Difference in means were tested using Welch's T-test.

Results

1. Mean baseline small bowel motility scores in CIPO patients was 0.19AU (range 0.1 to 0.25 ) and in controls 0.35AU (range 0.275 to 0.37 ) with a statistically significant difference of $0.17 \mathrm{AU}, \mathrm{P}=0.0026$ (CI 0.09 to 0.23 ).

2. The mean percent increase in small bowel motility scores in CIPO patients following noestigmine was 29\% (95\% CI from 19 to $50 \%$ ) and in controls $10 \%$ (range 0 to 34 ) with a statistically significant difference in groups response to neostigmine of $19 \%, \mathrm{P}=0.029$ (95\% CI from 4 to $40 \%$ ).

Conclusion This study demonstrated significant differences in both resting and cholinomimetic-induced global motility between CIPO patients and healthy controls. Despite marked bowel distension in the CIPO patients, motility appeared present but reduced compared to controls, and responded to provocation with neostigmine suggesting the bowel still exhibits the expected pro-kinetic effects following pharmacological stimulation. With just five patients this is a preliminary study, nevertheless initial results appear promising and support our ongoing investigation program.

\section{REFERENCE}

1 Menys et al. Global Assessment Radiol 2013;269(2):443-50

2 Odille et al. Qu, UKantitative assessment MRM 2012:68(3):783-93

Disclosure of Interest None Declared.

\section{PWE-182 DYSMOTILITY IN PARKINSON'S DISEASE CORRELATES TO GUT SYMPTOMS: FINDINGS OF A WIRELESS MOTILITY CAPSULE STUDY}

${ }^{1} \mathrm{SK}$ Butt ${ }^{*},{ }^{1} \mathrm{R}$ Leung, ${ }^{2} \mathrm{~A}$ Bala, ${ }^{2} \mathrm{~K}$ Bhatia, ${ }^{1} \mathrm{~A}$ Raeburn, ${ }^{1} \mathrm{~A}$ Emmanuel. ${ }^{1}$ Gastroenterology, UCL London, UK; ${ }^{2}$ Neurology, National Hospital for Neurology and Neurosurgery, London, UK

10.1136/gutjnl-2014-307263.442 